\title{
Reduced volume of the nucleus accumbens in heroin addiction
}

\author{
Christian L. Seifert · Stefano Magon · Till Sprenger · Undine E. Lang • \\ Christian G. Huber $\cdot$ Niklaus Denier $\cdot$ Marc Vogel $\cdot$ André Schmidt $\cdot$ \\ Ernst-Wilhelm Radue $\cdot$ Stefan Borgwardt $\cdot$ Marc Walter
}

Received: 3 July 2014 / Accepted: 24 November 2014 / Published online: 3 December 2014

(C) Springer-Verlag Berlin Heidelberg 2014

\begin{abstract}
The neural mechanisms of heroin addiction are still incompletely understood, even though modern neuroimaging techniques offer insights into disease-related changes in vivo. While changes on cortical structure have been reported in heroin addiction, evidence from subcortical areas remains underrepresented. Functional imaging studies revealed that the brain reward system and particularly the nucleus accumbens (NAcc) play a pivotal role in the pathophysiology of drug addiction. The aim of this study was to investigate whether there was a volume difference of the NAcc in heroin addiction in comparison to healthy controls. A further aim was to correlate subcortical volumes with clinical measurements on negative affects in addiction. Thirty heroin-dependent patients under maintenance treatment with diacetylmorphine and twenty healthy controls underwent structural MRI scanning at 3T.
\end{abstract}

C. L. Seifert · U. E. Lang · C. G. Huber · N. Denier · M. Vogel · A. Schmidt $\cdot$ S. Borgwardt $\cdot$ M. Walter Department of Psychiatry, University Psychiatric Clinics (UPK) of Basel, Basel, Switzerland

C. L. Seifert $(\bowtie)$

Department of Neurology, Technische Universität München, Ismaninger Str. 22, 81675 Munich, Germany

e-mail: c.seifert@1rz.tu-muenchen.de

S. Magon · T. Sprenger

Department of Neurology, University Hospital of Basel, Basel, Switzerland

T. Sprenger

Division of Neuroradiology, University Hospital of Basel, Basel, Switzerland

T. Sprenger $\cdot$ A. Schmidt $\cdot$ E.-W. Radue $\cdot$ S. Borgwardt Medical Image Analysis Centre, University Hospital of Basel, Basel, Switzerland
Subcortical segmentation analysis was performed using FMRIB's Integrated Registration and Segmentation Tool function of FSL. The State-Trait Anxiety Inventory and the Beck Depression Inventory were used to assess trait anxiety and depressive symptoms, respectively. A decreased volume of the left NAcc was observed in heroin-dependent patients compared to healthy controls. Depression score was negatively correlated with left NAcc volume in patients, whereas a positive correlation was found between the daily opioid dose and the volume of the right amygdala. This study indicates that there might be structural differences of the NAcc in heroin-dependent patients in comparison with healthy controls. Furthermore, correlations of subcortical structures with negative emotions and opioid doses might be of future relevance for the investigation of heroin addiction.

Keywords Heroin addiction - Diacetylmorphine ·

Subcortical · Grey matter changes · Nucleus accumbens

\section{Introduction}

Drug addiction including heroin dependence was investigated in a number of neuroimaging studies, which have repeatedly shown that drug-induced changes of brain structure and function have contributed to impairments in cognitive and emotional processes [4, 10, 24]. The neurobiology of disrupted self-control in drug dependence includes especially the brain reward system [involving the nucleus accumbens (NAcc), the pallidum and the striatum], but also memory and learning (including the amygdala and hippocampus), cognitive control [e.g. prefrontal cortex (PFC) and dorsal anterior cingulate cortex (ACC)], and motivation/drive and salience evaluation [e.g. orbital frontal cortex 
$(\mathrm{OFC})]$. Structural imaging studies related to heroin addiction traditionally used voxel-based morphometry (VBM) in the past. Liu et al. [21] published a VBM study, in which heroin-dependent patients showed reduced grey matter volumes in the right PFC, ACC and the left supplementary motor area when compared to healthy controls. Furthermore, another VBM study demonstrated a correlation of the duration of heroin use with decreased grey matter densities in prefrontal, temporal and cingulate cortices [58]. A further VBM study by Lyoo et al. [22] confirmed that opioiddependent subjects display grey matter decreases in prefrontal and temporal cortices. Of note, diffusion tensor and multimodal imaging studies also showed changes in white matter volumes of bilateral frontal sub-gyral regions, right precentral and left cingulate, and interestingly, the extent and severity of white matter integrity deficits in heroin addiction have been associated with the length of heroin dependency [20, 32, 40]. Functional MRI during the injection of diacetylmorphine resulted in a higher BOLD-activation in the OFC in comparison with the injection of placebo in a recent study by our group [50]. Functional connectivity and default mode network studies in chronic heroin users showed increased functional connectivity between the NAcc and the ventral/rostral ACC and OFC, respectively, and between the amygdala and OFC, while reduced functional connectivity have been observed between the PFC and OFC and between the PFC and ACC [23, 24, 33, 56]. Although animal studies support the key role of the NAcc in heroin addiction [16, 44], previous imaging studies using whole brain voxel-wise approaches such as VBM to analyse structural MRI have failed to identify structural changes in the NAcc. At least one multimodal neuroimaging study revealed a decreased volume of the amygdala in ten opioid-addicted patients in comparison with healthy controls, but no change in the NAcc [46].

Recently, we have found that acute heroin injection reduced negative emotions in heroin-dependent patients [4], while it also attenuated the amygdala response to fearful faces [39]. These studies might help to better understand how prolonged drug-taking behaviour is maintained through negative reinforcement mechanisms in anxiousdepressive heroin addicts. However, the influences of anxiety and depression on structural brain changes in heroin dependence remain unclear.

The crucial brain reward neurotransmitter activated by addictive drugs is dopamine. Despite different mechanisms, all abused drugs increase the dopamine transmission within the NAcc [28].

Distinctive profiles of gene expression in the human NAcc have been associated with heroin abuse [1]. Beneath basic research on reward and the NAcc in addiction, recent fMRI studies were able to demonstrate that craving correlated with the activation of the NAcc in heroin-addicted patients $[17,18]$. In the present study, we aimed to examine whether the volume of the NAcc differed between heroindependent patients and healthy controls using an automatic subcortical segmentation method.

Furthermore, we aimed to correlate subcortical volumes with clinical scores on trait anxiety and depression to investigate pathophysiological relationships between subcortical volumes, opioid doses and psychopathological hallmarks of behaviour.

\section{Methods}

This study is a sub-analysis of a randomized, placebo-controlled, crossover trial. Detailed information on the study protocol and other analyses has already been published [11, $39,50,51]$. After a detailed explanation of all study procedures, every subject provided written informed consent. The study was approved by the local ethics committee, and the study has been registered in http://clinicaltrials.gov with the ID NCT01174927. The study was carried out in accordance with the declaration of Helsinki.

\section{Participants}

Thirty patients who met the DSM-IV diagnostic criteria for opioid dependence were recruited from the Psychiatric Hospital of the University of Basel (Switzerland). Participants were instructed to abstain from illicit drug use other than prescribed heroin for the duration of the study, from alcohol intake for $72 \mathrm{~h}$ and from tobacco consume for $2 \mathrm{~h}$ before scanning. All participants were cigarette smokers. Inclusion criteria were age older than 18 years, history of intravenous heroin dependence, on current heroin-assisted treatment for at least 6 months and unchanged dose during the previous 3 months. Exclusion criteria were a positive alcohol breathalyzer test and severe additional physical, neurological or psychiatric disorder, including other comorbid substance dependence. History of heroin and other illicit substance use was assessed with the semi-structured interview according to ICD-10 criteria. All patients had at least two in-patient detoxification treatments for heroin dependence, an established opioid substitution, and participated in the standardized heroin-assisted treatment programme (JANUS, University of Basel, Switzerland). This includes the prescription of diacetylmorphine and is established for severe heroin dependence in Switzerland and is proven to be effective [30]. The healthy controls were screened using a semi-structured clinical interview to exclude psychiatric or physical illness or a family history of psychiatric illness. Controls that have ever used any other illicit psychotropic drug other than cannabinoids or fulfilled the criteria for alcohol dependence (who consumed $>20 \mathrm{~g}$ 
alcohol per day) or who had any psychiatric, neurological or severe medical illness history were also excluded. Cannabis and cocaine use was determined by urine test.

Controls were recruited from the general population by advertisement in the same geographical area.

Image acquisition of MRI data

For anatomical imaging, a 3D T1-weighted MPRAGE sequence was applied with $1 \times 1 \times 1 \mathrm{~mm}^{3}$ isotropic resolution and with inversion time of $1,000 \mathrm{~ms}$, repetition time of $8 \mathrm{~ms}$ and echo time of $3.4 \mathrm{~ms}$. We used a 3T scanner, Siemens Magnetom Verio, Siemens Healthcare, Erlangen, Germany. All scans were reviewed for significant radiological abnormalities by an experienced neuroradiologist.

\section{MRI data analysis, FSL/FIRST}

Volumes of subcortical structures were estimated on 3D T1-weighted images using FIRST, the FMRIB's Integrated Registration and Segmentation Tool function of FSL (FMRIB Software Library) FSL/FIRST [7, 31]. FIRST is implemented in the freely available FSL package [55] and is a model-based automated registration/segmentation tool based on a training set of 336 images. The segmentation model uses a Bayesian Appearance Model, based on multivariate Gaussian assumptions, measuring the probabilistic relationships between shape and grey matter intensity and was recently developed for subcortical structural volume analysis [31]. This method of automatic volumetry has clear advantages compared to VBM and manual segmentation. VBM has shown its value in comparing groups of subjects in patterns of atrophy, but is prone to registration artefacts in the deep grey matter (DGM) [5]. Compared to manual segmentation, FIRST has the advantage that its segmentation is based on voxel intensities, while in manual segmentation, the contrast differences can be difficult to detect visually. Furthermore, since an automatic method is used, the researcher bias in segmentation is very low [8]. Segmented structures via FIRST included: hippocampus, amygdala, caudate, putamen, thalamus, NAcc, the pallidum and the brainstem including fourth ventricle. The deformable surfaces of deep GM structures were used to automatically parameterize volumetric labels in terms of meshes. The normalized intensities along the surface of meshes were sampled and modelled. After the automated segmentation by the software, the segmentations were visually checked one by one and confirmed for the proper segmentation of all subcortical structures. An example of the segmentation is presented in Fig. 1, showing representative slices of the reported subcortical brain areas.

The absolute volumes of thalamus, hippocampus, amygdala, caudate, putamen, pallidum and NAcc were then estimated. Further, statistical comparisons between groups were performed using SPSS software (IBM SPSS Statistics for Windows, Version 19). The vertex-wise analysis was conducted for the NAcc to detect differences on the shape of the surface of the.

Moreover, the native brain volume (nBV) was computed for each subjects using the high-resolution T1-weighted images with the fully automated tool Structural Image Evaluation using Normalization of Atrophy for cross-sectional studies (SIENAX version 2.6) [41].

\section{Clinical data assessments}

The Beck Depression Inventory (BDI), a widely used 21-question multiple-choice self-report inventory, was applied to assess depressive symptoms [2]. The BDI is also proven to be valid in opioid addiction [13]. The Spielberger State-Trait Anxiety Inventory (STAI) is one of the most widely known self-report scales to measure anxiety and has been used extensively in the past decades as a research and clinical instrument [42]. The STAI consists of two scales: one scale measures how one generally feels (trait) and one scale measures how one feels at the moment (state). The trait anxiety can be quantified as a comparatively stable personality trait and was performed in all subjects. The daily dose of opioids has been registered from the patients' records. As some patients were on diacetylmorphine plus methadone or oral morphine, we translated the doses in diacetylmorphine in accordance with established clinical recommendations as used in other studies [47]. Craving was assessed using the 45-item Heroin Craving Questionnaire [45] which measures positive and negative aspects of craving on five theory-derived 9-item scales. The corresponding scale measures the desire to use heroin.

\section{Statistics}

Mean regional GM volumetric differences between the study groups were investigated, using the Student's $t$ test. A $p$ value $<0.05$ was considered to represent a significant difference. Moreover, an additional ANCOVA was performed including age, gender, cocaine abuse and native brain volume as covariate. A rank analysis of covariates [35] was performed when the statistical model did not meet the assumptions for parametric ANCOVA.

Spearman rank correlation analysis was used to assess the relationships between regional GM volumes and clinical parameters such as daily opioid dose and BDI and STAI/ Trait anxiety scores. Correlation analysis was corrected for multiple comparisons using Bonferroni correction. Twelve correlation analyses were performed, and $p<0.0042$ was considered as $p<0.05$, Bonferroni-corrected. 


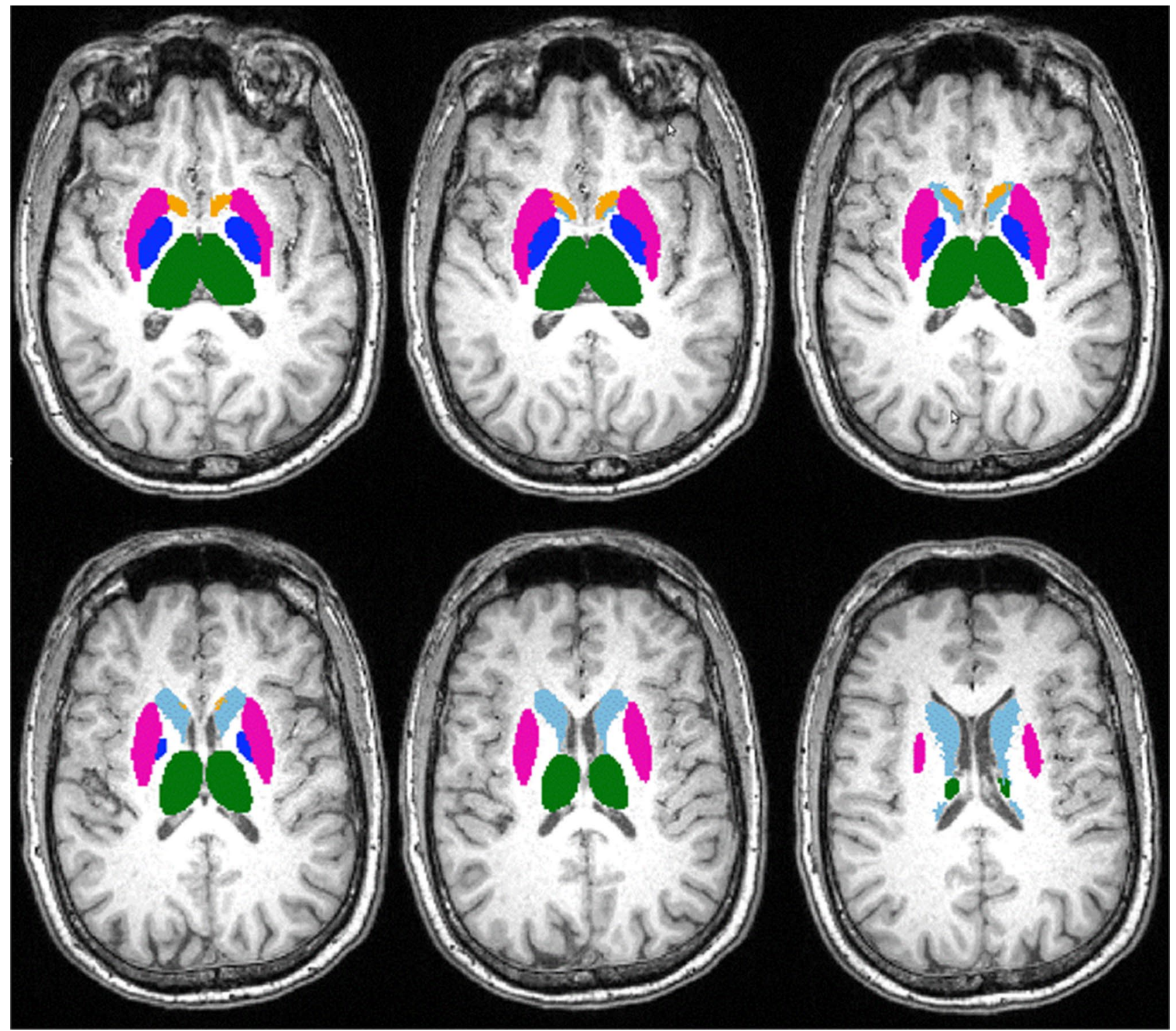

Fig. 1 Example of segmentation by FIRST analysis in a single subject (heroin-addicted patient). Slices are presented in radiological convention showing the original T1 map and an overlay of segmented

\section{Results}

The main baseline characteristics of heroin-addicted patients and healthy controls are summarized in Table 1 . Patients were aged 23-58 years (average age $=41.1$, $\mathrm{SD}=6.4)$, and all patients had been in heroin-assisted treatment for 6.7 years on average $(\mathrm{SD}=4.5)$. Heroinmaintained patients showed significantly higher STAI and BDI scores than healthy controls. Whereas no healthy control subject used cocaine, $53 \%$ of patients did. structures from lower slices to more cranial slices from the left to the right. Colours indicate the different structures: blue pallidum, green thalamus, orange NAcc, light blue Ncl. Caudatus, purple putamen
FSL-FIRST analysis

Between-group comparison revealed a significant difference $(p=0.049)$ in the left NAcc with a decrease in the volume in the group of heroin-addicted patients (patients: $458.9 \pm 81.8 \mathrm{~mm}^{3}$, controls; $516.7 \pm 116.4 \mathrm{~mm}^{3}$ ). The rank ANCOVA showed a significant difference in the left NAcc as well $(U=131, p<0.001)$. No significant differences were found in other subcortical areas. The vertex-wise shape analysis did not yield any significant local shape differences in the NAcc. An example of the 
Table 1 Baseline data of patients and control group: comparison by Student's $t$ test with $p$ values

$S D$ standard deviation, STAI State-Trait Anxiety Inventory, $B D I$ Beck Depression Inventory, n.s. not significant

\begin{tabular}{llll}
\hline & Patients $(N=30)$ & Controls $(N=20)$ & $t$ test $/ p$ values \\
\hline Age mean (SD) & $41.1(6.4)$ & $40.2(10.9)$ & n.s. \\
Male:female ratio in \% & $70: 30$ & $70: 30$ & n.s. \\
Duration of heroin addiction in years (SD) & $20.7(6.4)$ & - & - \\
Daily dose of diacetylmorphine in mg & $325.3(131.4)$ & - & - \\
Duration of maintenance with DAM in years (SD) & $6.7(4.5)$ & - & - \\
Smoking in \% & 100 & 100 & n.s. \\
Number of cigarettes per day (SD) & $20.4(9.1)$ & $11.5(8.2)$ & $<0.001$ \\
Use of alcohol in \% & 0 & 0 & n.s. \\
Use of cannabinoids in \% & 30 & 25 & n.s. \\
Use of cocaine in \% & 53 & 0 & $<0.001$ \\
STAI Trait score (SD) & $42.9(8.8)$ & $31.6(6.4)$ & $<0.001$ \\
STAI State score (SD) & $42.6(9.8)$ & $32.9(8.5)$ & $=0.001$ \\
BDI (SD) & $15.2(8.1)$ & $2.2(2.8)$ & $<0.001$ \\
HCQ (desire to use heroin) & $5.29(1.32)$ & - & - \\
HCQ (intentions and plans to use heroin) & $5.34(1.01)$ & - & - \\
HCQ (anticipation of positive outcome) & $5.37(1.01)$ & - & - \\
HCQ (relief from withdrawal/dysphoria) & $4.91(1.17)$ & - & - \\
HCQ (lack of control over use) & $4.93(1.05)$ & - & - \\
HCQ (lack of control over use) & $4.93(1.05)$ & - & - \\
\hline
\end{tabular}

Table 2 Correlation analysis in heroin-addicted patients with clinical data

\begin{tabular}{|c|c|c|c|c|c|}
\hline & Correlation parameter & Amyg left & NAcc left & Amyg right & NAcc right \\
\hline Daily dose of diacetylmorphine in $\mathrm{mg}$ & Corr. coefficient (Spearmen) $\rho$ & $0.535^{*} p=0.002$ & -0.108 & 0.101 & -0.060 \\
\hline STAI-trait sumscore & Corr. coefficient (Spearmen) $\rho$ & 0.109 & -0.416 & -0.191 & -0.276 \\
\hline BDI & Corr. coefficient (Spearmen) $\rho$ & 0.030 & $-0.525^{*} p=0.003$ & -0.243 & -0.231 \\
\hline
\end{tabular}

Spearmen correlation was used

NAcc Nucleus accumbens; Amyg Amygdala; STAI State-Trait Anxiety Inventory; BDI Beck Depression Inventory

* Significant correlation (corrected for multiple comparison)

FIRST segmentation in a single patient is presented in Fig. 1.

\section{Correlation analysis}

Significant correlations occurred between daily doses of opioids and the volume of the left amygdala $(p=0.002)$, and a significant negative correlation has been observed between the left NAcc and the BDI $(p=0.003)$ in patients, but not in healthy controls. The correlation analysis between clinical parameters and subcortical volumes of the amygdala and the NAcc can be reviewed in Table 2. The corresponding graphs are displayed in Fig. 2, showing only significant correlations in patients. In healthy controls, no significant correlations have been observed in our analysis. Correlation analysis with heroin craving questionnaire did not show any significant correlations.

\section{Discussion}

To the best of our knowledge, this is the first study demonstrating a structural volume decrease in the left NAcc in heroin-addicted patients compared with healthy controls using the segmentation technique FIRST. The NAcc is part of the reward circuitry involved in drug-seeking behaviours [7]. The NAcc discriminates the motivational value of conditioned stimuli, and it predetermines behaviour for unconditioned stimuli, including the repeated use of addictive drugs [27]. In contrast to a previous study showing reduced volume in the bilateral amygdala in a small sample of ten opioid-dependent patients [46], we found no significant changes in the amygdala volume. The fact that we did not observe such differences in the amygdala might be partly explained by differences in the number of patients between the studies and the different kind of diseases, which were investigated (heroin addiction versus prescription opioid dependence). The volume reduction in the 

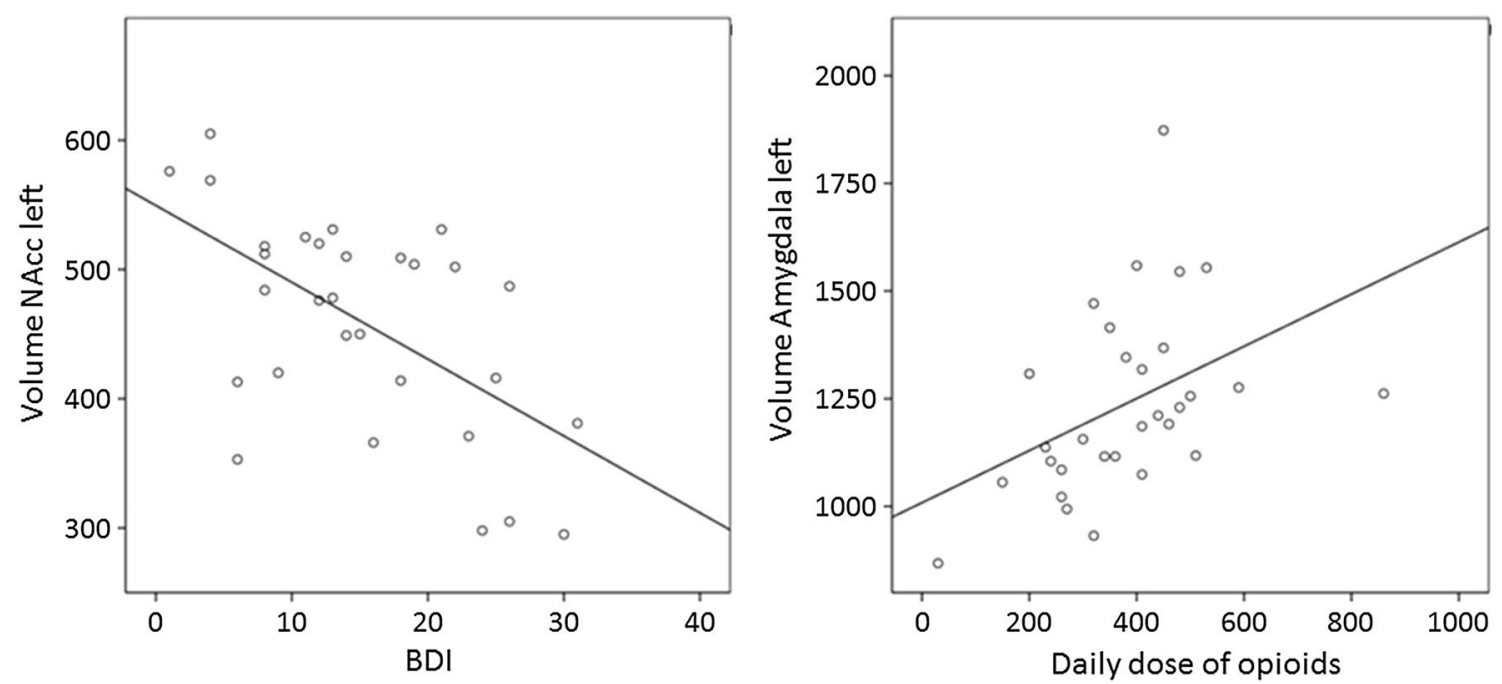

Fig. 2 Significant correlations (after correction for multiple comparisons) of volumes of the left NAcc and the left amygdala ( $y$-axis) with clinical parameters ( $x$-axis) in patients with heroin addiction

left NAcc was not shown to be a matter of the surface/shape as indicated by the shape analysis. The reason for that might be either that the structure is too small to show significant differences in the local shape or that the volume difference is not based on volume loss on the surface but in the depth of the NAcc. That could be a result of different binding densities of mu-opioid receptors within the NAcc, which have been shown to have a higher binding in the core of the NAcc and the dorsomedial NAcc compared with the ventral NAcc shell [36]. In heroin addiction, reduced frontal and cingulate grey matter volumes were mostly reported in former VBM studies [19, 57]. Moreover, these volume reductions have been shown to be correlated with impulsivity behaviour in heroin addiction [34]. Some of the alterations observed in VBM studies even seem to be reversible after abstinence [53]. It is imaginable that the decrease observed in the NAcc in our study could be a consequence of the chronic intake of opioids. From animal studies, it is well known that morphine alters the structure of neurons in the NAcc and neocortex: in rats, prior exposure to morphine decreases the complexity of dendritic branching and the number of dendritic spines on medium spiny neurons in the shell of the NAcc and on pyramidal cells in the prefrontal and parietal cortex [37]. In animal studies of other addictive states, Wheeler et al. [54] reported in animal studies with mice exposed to cocaine during adolescence a bilateral decrease in the NAcc. A second hypothesis might be that the reduction in the left NAcc is prior to the development of heroin addiction. At least anhedonia has been linked to decreased NAcc volumes [49] and deep brain stimulation of NAcc can decrease ratings of anxiety and depression [3]. Therefore, increased depression and anxiety might occur in humans with decreased NAcc volumes, leading secondly to an increased risk of heroin consumption and higher doses of opioids. The interaction between addiction and depressive symptoms might be mainly influenced by the reward system. In line with this hypothesis, it has been shown that mood disorders are characterized in part by profound deficits in reward-related behaviour. A recent study has identified important structural and functional alterations within the brain's reward circuitry-particularly in the ventral tegmental area-NAcc pathway-that are associated with symptoms such as anhedonia and aberrant reward-associated perception and memory [38].

One could furthermore ask about the lateralization with respect to the result of volume decrease in the left NAcc presented in our study. This point is difficult to interpret, but what is known from a clinical observational study in China, which investigated physiological and psychological reactions to electric stimulation of the amygdaloid nucleus and the NAcc in heroin patients, is that stimulation of either the amygdala or the NAcc induces euphoria similar to heroin-induced euphoria, especially when the left amygdala or left NAcc was stimulated [9]. This is in line with our results, and we hypothesize that via chronic intake of opioids, the "overstimulation" of the NAcc by exogenous opioids could lead to a volume decrease starting in the left NAcc. A more simple explanation of the lateralization observed in our results might be an insufficient number of subjects included. Given the fact that the NAcc is quite a small structure and the variance in segmentation studies of this structure is relatively broad [29] and from a statistically point of view, it is imaginable that the observed effects in our study might be even greater. In our correlation analysis, we found that depression scores were negatively correlated with left NAcc volume in patients, but not in healthy controls, i.e. patients with depressive symptoms showed a lower NAcc volume. 
We can argue that heroin-dependent patients with depressive symptoms may use the drug mainly from negative reinforcement [39]. Negative reinforcement can be defined as the process by which removal of an aversive stimulus such as a negative emotional state increases the probability of drug-taking behaviour [14]. We already could demonstrate that acute heroin administration was associated with reduced stress and negative emotions [39, $51,52]$. One could argue that the NAcc-characterized as a key system in drug reward-is mainly involved in the positive reinforcement of drug-taking behaviour via a dopamine increase in the NAcc [48]. Our results could contribute a clinical marker to distinguish subgroups of patients by the volume of the NAcc with respect to depressive symptoms. This seems relevant as pheno- and genotyping of different subtypes of heroin addiction and related behaviours have recently attracted high attention [43]. Whether the negative correlation of the NAcc with depression we observed is specific for heroin addiction or might be caused by depression itself without a relation to addiction must be investigated. Numerous studies have shown no change in NAcc volume in major depression $[6,12]$. Both cited studies used older classical semi-automatic and non-standardized volumetric approaches, and therefore, it is not easy to exclude a methodological bias in these studies. But, interestingly, functional activity of the NAcc (and the ventral striatum as a whole) was shown to be reduced in major depressive disorders [26]. One study investigated the influence of the antidepressant duloxetine on subcortical volumes of firstepisode patients with major depressive disorder and panic disorder and showed modest increases in volumes of the bilateral NAcc amongst others [15]. Interestingly, we also found a correlation between amygdala volume and the injected heroin dose. This could also be interpreted in the context of negative emotional processing in heroin dependence. In a recent fMRI study, our group was also able to demonstrate acute effects heroin on the amygdala activity with negative emotional processing using response to fearful faces [39]. Although we found no difference in the volume of the amygdala between patients and controls, one might speculate that the correlation of absolute volume of the amygdala with the injected heroin might be associated with increased anxiety, since increased volumes of the amygdala have also been shown in anxiety disorders [25].

\section{Limitations}

There are some limitations that have to be considered in the present study:

The major limitation of this study is that the result of the FIRST analysis is statistically weak and will not be significant after correction for multiple comparisons.
Nevertheless, in our opinion, this result is supported by former studies and hypotheses, which showed a key role of the NAcc in heroin addiction. Therefore, our result could lead future studies, which might be able to confirm the structural difference in the NAcc. Furthermore, the improvement of subcortical segmentation tools will help to clarify the results of our study in future.

Minor limitations of this study are that healthy controls and patients may differ in several variables such as educational level, level of employment or polysubstance use such as consumption of nicotine and cocaine, what may have led to confounding our results. Even though we excluded patients with additional psychiatric diagnoses, we cannot definitely exclude that heroin-addicted patients might suffer from other "sub-threshold symptoms" in detail depressive and anxiety symptoms [4] which might lead to bias our results.

Moreover, our patients were recruited from a population which mainly consisted of individuals with long-standing polysubstance use. Patients in heroin-maintenance programme are usually in severe status of heroin dependence and may have more comorbidity mental health problems and polydrug use. Although this problem is virtually inevitable when chronic heroin-dependent individuals are examined, it may have biased the results. The findings may thus not apply to all groups of heroin users and/or maintenance patients.

\section{Conclusion}

In conclusion, we were able to show a volume decrease in the left NAcc volume in heroin-addicted patients on maintenance treatment with diacetylmorphine. We could further show that depression scores correlated negatively with the volume of the left NAcc. Our results suggest that smaller volume of the left NAcc in patients with a depressive phenotype constitutes a biological substrate of opioid addiction and therefore could possibly be useful in further studies investigating risk prediction.

Acknowledgments This study was supported by the Swiss National Science Foundation (SNSF) (32003B-127544). We would like to acknowledge the infrastructural support of the Medical Image Analysis Centre, University Hospital Basel.

Conflict of interest Till Sprenger served on advisory boards for Mitsubishi Pharma, Eli Lilly, Genzyme, Novartis, Biogen and Allergan. The other authors declare no conflict of interest.

\section{References}

1. Albertson DN, Schmidt CJ, Kapatos G, Bannon MJ (2006) Distinctive profiles of gene expression in the human nucleus accumbens associated with cocaine and heroin abuse. Neuropsychopharmacology 31:2304-2312 
2. Beck AT, Erbaugh J, Ward CH, Mock J, Mendelsohn M (1961) An inventory for measuring depression. Arch Gen Psychiatry 4:561

3. Bewernick BH, Hurlemann R, Matusch A, Kayser S, Grubert C, Hadrysiewicz B, Axmacher N, Lemke M, Cooper-Mahkorn D, Cohen MX, Brockmann H, Lenartz D, Sturm V, Schlaepfer TE (2010) Nucleus accumbens deep brain stimulation decreases ratings of depression and anxiety in treatment-resistant depression. Biol Psychiatry 67:110-116

4. Blum J, Gerber H, Gerhard U, Schmid O, Petitjean S, RiecherRossler A, Wiesbeck GA, Borgwardt SJ, Walter M (2013) Acute effects of heroin on emotions in heroin-dependent patients. Am J Addict 22:598-604

5. Bookstein FL (2001) "Voxel-based morphometry" should not be used with imperfectly registered images. Neuroimage 14:1454-1462

6. Bremner JD, Narayan M, Anderson ER, Staib LH, Miller HL, Charney DS (2000) Hippocampal volume reduction in major depression. Am J Psychiatry 157:115-118

7. Carlezon WA Jr, Thomas MJ (2009) Biological substrates of reward and aversion: a nucleus accumbens activity hypothesis. Neuropharmacology 56(Suppl 1):122-132

8. de Jong LW, van der Hiele K, Veer IM, Houwing JJ, Westendorp RG, Bollen EL, de Bruin PW, Middelkoop HA, van Buchem MA, van der Grond J (2008) Strongly reduced volumes of putamen and thalamus in Alzheimer's disease: an MRI study. Brain 131:3277-3285

9. Fang J, Gu JW, Yang WT, Qin XY, Hu YH (2012) Clinical observation of physiological and psychological reactions to electric stimulation of the amygdaloid nucleus and the nucleus accumbens in heroin addicts after detoxification. Chin Med J 125:63-66

10. Franken IH, Kroon LY, Wiers RW, Jansen A (2000) Selective cognitive processing of drug cues in heroin dependence. J Psychopharmacol 14:395-400

11. Gerber H, Borgwardt SJ, Schmid O, Gerhard U, Joechle W, Riecher-Rossler A, Wiesbeck GA, Walter M (2012) The impact of diacetylmorphine on hypothalamic-pituitary-adrenal axis activity and heroin craving in heroin dependence. Eur Addict Res $18: 116-123$

12. Hannestad J, Taylor WD, McQuoid DR, Payne ME, Krishnan KR, Steffens DC, Macfall JR (2006) White matter lesion volumes and caudate volumes in late-life depression. Int J Geriatr Psychiatry 21:1193-1198

13. Hesse M (2006) The Beck Depression Inventory in patients undergoing opiate agonist maintenance treatment. Br J Clin Psychol 45:417-425

14. Koob GF (2009) Dynamics of neuronal circuits in addiction: reward, antireward, and emotional memory. Pharmacopsychiatry 42(Suppl 1):S32-S41

15. Lai CH, Wu YT (2011) Duloxetine's modest short-term influences in subcortical structures of first episode drug-naive patients with major depressive disorder and panic disorder. Psychiatry Res 194:157-162

16. LaLumiere RT, Kalivas PW (2008) Glutamate release in the nucleus accumbens core is necessary for heroin seeking. J Neurosci 28:3170-3177

17. Li Q, Li W, Wang H, Wang Y, Zhang Y, Zhu J, Zheng Y, Zhang D, Wang L, Li Y, Yan X, Chang H, Fan M, Li Z, Tian J, Gold MS, Wang W, Liu Y (2014) Predicting subsequent relapse by drugrelated cue-induced brain activation in heroin addiction: an eventrelated functional magnetic resonance imaging study. Addict Biol. doi:10.1111/adb.12182

18. Li Q, Wang Y, Zhang Y, Li W, Yang W, Zhu J, Wu N, Chang H, Zheng Y, Qin W, Zhao L, Yuan K, Liu J, Wang W, Tian J (2012) Craving correlates with mesolimbic responses to heroin-related cues in short-term abstinence from heroin: an event-related fMRI study. Brain Res 1469:63-72

19. Liu H, Hao Y, Kaneko Y, Ouyang X, Zhang Y, Xu L, Xue Z, Liu $Z$ (2009) Frontal and cingulate gray matter volume reduction in heroin dependence: optimized voxel-based morphometry. Psychiatry Clin Neurosci 63:563-568

20. Liu H, Li L, Hao Y, Cao D, Xu L, Rohrbaugh R, Xue Z, Hao W, Shan B, Liu Z (2008) Disrupted white matter integrity in heroin dependence: a controlled study utilizing diffusion tensor imaging. Am J Drug Alcohol Abuse 34:562-575

21. Liu X, Matochik JA, Cadet JL, London ED (1998) Smaller volume of prefrontal lobe in polysubstance abusers: a magnetic resonance imaging study. Neuropsychopharmacology 18:243-252

22. Lyoo IK, Pollack MH, Silveri MM, Ahn KH, Diaz CI, Hwang J, Kim SJ, Yurgelun-Todd DA, Kaufman MJ, Renshaw PF (2006) Prefrontal and temporal gray matter density decreases in opiate dependence. Psychopharmacology 184:139-144

23. Ma N, Liu Y, Fu XM, Li N, Wang CX, Zhang H, Qian RB, Xu HS, Hu X, Zhang DR (2011) Abnormal brain default-mode network functional connectivity in drug addicts. PLoS One 6:e16560

24. Ma N, Liu Y, Li N, Wang CX, Zhang H, Jiang XF, Xu HS, Fu XM, Hu X, Zhang DR (2010) Addiction related alteration in resting-state brain connectivity. Neuroimage 49:738-744

25. Machado-de-Sousa JP, de Lima Osorio F, Jackowski AP, Bressan RA, Chagas MH, Torro-Alves N, Depaula AL, Crippa JA, Hallak JE (2014) Increased amygdalar and hippocampal volumes in young adults with social anxiety. PLoS One 9:e88523

26. Mayberg HS, Brannan SK, Tekell JL, Silva JA, Mahurin RK, McGinnis S, Jerabek PA (2000) Regional metabolic effects of fluoxetine in major depression: serial changes and relationship to clinical response. Biol Psychiatry 48:830-843

27. Meredith GE, Baldo BA, Andrezjewski ME, Kelley AE (2008) The structural basis for mapping behavior onto the ventral striatum and its subdivisions. Brain Struct Funct 213:17-27

28. Nestler EJ, Malenka RC (2004) The addicted brain. Sci Am 290:78-85

29. Nugent AC, Luckenbaugh DA, Wood SE, Bogers W, Zarate CA Jr, Drevets WC (2012) Automated subcortical segmentation using first: test-retest reliability, interscanner reliability, and comparison to manual segmentation. Hum Brain Mapp 34:2313-2329

30. Oviedo-Joekes E, Brissette S, Marsh DC, Lauzon P, Guh D, Anis A, Schechter MT (2009) Diacetylmorphine versus methadone for the treatment of opioid addiction. N Engl J Med 361:777-786

31. Patenaude B, Smith SM, Kennedy DN, Jenkinson M (2011) A bayesian model of shape and appearance for subcortical brain segmentation. Neuroimage 56:907-922

32. Qiu Y, Jiang G, Su H, Lv X, Zhang X, Tian J, Zhuo F (2013) Progressive white matter microstructure damage in male chronic heroin dependent individuals: a DTI and TBSS study. PLoS One 8:e63212

33. Qiu YW, Han LJ, Lv XF, Jiang GH, Tian JZ, Zhuo FZ, Su HH, Lin CL, Zhang XL (2011) Regional homogeneity changes in heroin-dependent individuals: resting-state functional MR imaging study. Radiology 261:551-559

34. Qiu YW, Jiang GH, Su HH, Lv XF, Tian JZ, Li LM, Zhuo FZ (2013) The impulsivity behavior is correlated with prefrontal cortex gray matter volume reduction in heroin-dependent individuals. Neurosci Lett 538:43-48

35. Quade D (1967) Rank analysis of covariance. J Am Stat Assoc 62:1187-1200

36. Resendez SL, Dome M, Gormley G, Franco D, Nevarez N, Hamid AA, Aragona BJ (2013) Mu-opioid receptors within subregions of the striatum mediate pair bond formation through parallel yet distinct reward mechanisms. J Neurosci 33:9140-9149 
37. Robinson TE, Kolb B (1999) Morphine alters the structure of neurons in the nucleus accumbens and neocortex of rats. Synapse 33:160-162

38. Russo SJ, Nestler EJ (2013) The brain reward circuitry in mood disorders. Nat Rev Neurosci 14:609-625

39. Schmidt A, Borgwardt S, Gerber H, Wiesbeck GA, Schmid O, Riecher-Rossler A, Smieskova R, Lang UE, Walter M (2014) Acute effects of heroin on negative emotional processing: relation of amygdala activity and stress-related responses. Biol Psychiatry 76:289-296

40. Shen Y, Wang E, Wang X, Lou M (2012) Disrupted integrity of white matter in heroin-addicted subjects at different abstinent time. J Addict Med 6:172-176

41. Smith SM, Jenkinson M, Woolrich MW, Beckmann CF, Behrens TE, Johansen-Berg H, Bannister PR, De Luca M, Drobnjak I, Flitney DE, Niazy RK, Saunders J, Vickers J, Zhang Y, De Stefano N, Brady JM, Matthews PM (2004) Advances in functional and structural MR image analysis and implementation as FSL. Neuroimage 23(Suppl 1):S208-S219

42. Spielberger CD (1983) Manual for the State-Trait Anxiety Inventory (STAI). Santa Clara, PaloAlto

43. Sun J, Bi J, Chan G, Oslin D, Farrer L, Gelernter J, Kranzler HR (2012) Improved methods to identify stable, highly heritable subtypes of opioid use and related behaviors. Addict Behav 37:1138-1144

44. Szczytkowski JL, Fuchs RA, Lysle DT (2011) Ventral tegmental area-basolateral amygdala-nucleus accumbens shell neurocircuitry controls the expression of heroin-conditioned immunomodulation. J Neuroimmunol 237:47-56

45. Tiffany ST (1999) Cognitive concepts of craving. Alcohol Res Health 23:215-224

46. Upadhyay J, Maleki N, Potter J, Elman I, Rudrauf D, Knudsen J, Wallin D, Pendse G, McDonald L, Griffin M, Anderson J, Nutile L, Renshaw P, Weiss R, Becerra L, Borsook D (2010) Alterations in brain structure and functional connectivity in prescription opioid-dependent patients. Brain 133:2098-2114

47. Vogel M, Knopfli B, Schmid O, Prica M, Strasser J, Prieto L, Wiesbeck GA, Dursteler-Macfarland KM (2013) Treatment or "high": benzodiazepine use in patients on injectable heroin or oral opioids. Addict Behav 38:2477-2484

48. Volkow ND, Wang GJ, Fowler JS, Tomasi D, Telang F (2011) Addiction: beyond dopamine reward circuitry. Proc Natl Acad Sci USA 108:15037-15042
49. Wacker J, Dillon DG, Pizzagalli DA (2009) The role of the nucleus accumbens and rostral anterior cingulate cortex in anhedonia: integration of resting EEG, fMRI, and volumetric techniques. Neuroimage 46:327-337

50. Walter M, Denier N, Gerber H, Schmid O, Lanz C, Brenneisen R, Riecher-Rossler A, Wiesbeck GA, Scheffler K, Seifritz E, McGuire P, Fusar-Poli P, Borgwardt S (2014) Orbitofrontal response to drug-related stimuli after heroin administration. Addict Biol. doi:10.1111/adb.12145

51. Walter M, Gerber H, Kuhl HC, Schmid O, Joechle W, Lanz C, Brenneisen R, Schachinger H, Riecher-Rossler A, Wiesbeck GA, Borgwardt SJ (2013) Acute effects of intravenous heroin on the hypothalamic-pituitary-adrenal axis response: a controlled trial. J Clin Psychopharmacol 33:193-198

52. Walter M, Wiesbeck GA, Degen B, Albrich J, Oppel M, Schulz A, Schachinger H, Dursteler-MacFarland KM (2011) Heroin reduces startle and cortisol response in opioid-maintained heroindependent patients. Addict Biol 16:145-151

53. Wang X, Li B, Zhou X, Liao Y, Tang J, Liu T, Hu D, Hao W (2012) Changes in brain gray matter in abstinent heroin addicts. Drug Alcohol Depend 126:304-308

54. Wheeler AL, Lerch JP, Chakravarty MM, Friedel M, Sled JG, Fletcher PJ, Josselyn SA, Frankland PW (2013) Adolescent cocaine exposure causes enduring macroscale changes in mouse brain structure. J Neurosci 33:1797-1803

55. Woolrich MW, Jbabdi S, Patenaude B, Chappell M, Makni S, Behrens T, Beckmann C, Jenkinson M, Smith SM (2009) Bayesian analysis of neuroimaging data in fsl. Neuroimage 45:S173-S186

56. Yuan K, Qin W, Dong M, Liu J, Liu P, Zhang Y, Sun J, Wang W, Wang Y, Li Q, Yang W, Tian J (2010) Combining spatial and temporal information to explore resting-state networks changes in abstinent heroin-dependent individuals. Neurosci Lett 475:20-24

57. Yuan K, Qin W, Dong M, Liu J, Sun J, Liu P, Zhang Y, Wang W, Wang Y, Li Q, Zhao L, von Deneen KM, Liu Y, Gold MS, Tian J (2010) Gray matter deficits and resting-state abnormalities in abstinent heroin-dependent individuals. Neurosci Lett 482:101-105

58. Yuan Y, Zhu Z, Shi J, Zou Z, Yuan F, Liu Y, Lee TM, Weng X (2009) Gray matter density negatively correlates with duration of heroin use in young lifetime heroin-dependent individuals. Brain Cogn 71:223-228 